\title{
Gamma-Ray Astronomy with a Wide Field of View detector operated at Extreme Altitude in the Southern Hemisphere.
}

\author{
G. Di Sciascio*, S. Miozzi, R. Santonico \\ INFN - Rome Tor Vergata, Italy \\ E-mail: disciascio@roma2.infn.it \\ P. Montini \\ INFN - Rome La Sapienza, Italy \\ G. Piano, M. Tavani \\ INAF-IAPS, Rome, Italy
}

\begin{abstract}
The new generation of wide Field of View (FoV) EAS instruments (HAWC and LHAASO) are both in the Northern hemisphere and have access to a limited portion of the Galactic plane. A crucial part of the sky needs to be studied as well. The Southern hemisphere includes the Galactic Center, the Inner Galaxy, and includes a large number of extremely interesting sources (neutron stars, black holes, SNRs, and extra-galactic AGNs) relevant to study fundamental physics and high-energy particle acceleration phenomena. In this contribution we discuss the main drives for the design of a wide FoV gamma-ray telescope in the sub-TeV/TeV energy region.
\end{abstract}

35th International Cosmic Ray Conference - ICRC2017

10-20 July, 2017

Bexco, Busan, Korea

\footnotetext{
*Speaker.
} 


\section{Introduction}

High energy gamma ray (and neutrino) observations are, in principle, an essential probe of cosmic rays (hereafter CRs). In fact photons and neutrinos travel in straight lines, unperturbed by magnetic fields, and, unlike the charged CRs, point back to their sources, providing the direction of the CR accelerator. The integrated study of charged CRs, gamma rays and neutrinos is one of the most important and exciting fields in the so-called 'multi-messenger astronomy'.

Despite large progresses in building new detectors and in the analysis techniques, the key questions concerning origin, acceleration and propagation of CRs are still open. In particular, the identification of sources in our Galaxy able to accelerate particles up to PeV energies, the so-called 'PeVatrons', is still missing. In fact, even there is no doubt that galactic CRs are accelerated in SNRs, the capability of SNRs to accelerate CRs up to the knee of the spectrum and beyond, and to produce the content of CRs in our Galaxy, is still under debate.

Open problems in CR physics push the construction of new generation Extensive Air Shower (EAS) arrays to study at the same time photon- and charged-induced events. Their large field of view $(\mathrm{FoV})\left(\Omega_{\mathrm{FoV}} \sim \mathrm{O}(\mathrm{sr})\right)$ and high duty cycle $(\sim 100 \%)$ make this observational technique particularly suited to perform unbiased all-sky surveys (not simply of limited regions of the Galactic plane), to monitor the sky for the brightest transient emission from AGNs and GRBs, and to search for unknown transient phenomena. In addition a wide FoV detector allows to study extended $\gamma$-ray sources and to map the diffuse emission from the Galactic Plane.

In Fig. 1 the differential sensitivities to a Crab-like point gamma-ray source of different experiments/projects are compared. The new wide FoV $\gamma$-ray experiments under way in the coming years (HAWC and LHAASO) are located in the Northern hemisphere. A new EAS array to study the $\gamma$-sky of the Southern hemisphere above $100 \mathrm{GeV}$ with high sensitivity and to continuously monitor the Galactic Center and the Inner Galaxy should be a high priority. The expected sensitivity of the LATTES project [1] is also shown. LATTES will be constituted by a layer of RPCs on top of a water Cherenkov detector to exploit the added value of the combination of two different detectors. By operating the ARGO-YBJ experiment we demonstrated that RPCs can be safely operated at extreme altitudes for many years. The benefits in the use of RPCs in ARGO-YBJ were: (1) high efficiency detection of low energy showers (energy threshold $\sim 300 \mathrm{GeV}$ ) by means of the dense sampling of the central carpet; (2) unprecedented wide energy range investigated by means of the digital/charge read-outs $(\sim 300 \mathrm{GeV} \rightarrow 10 \mathrm{PeV})$; (3) good angular resolution and unprecedented details in the core region by means of the high granularity of the read-outs [2].

In this paper we discuss the main parameters which determine the sensitivity of a ground-based wide FoV gamma-ray telescope.

\section{Sensitivity to a $\gamma$-ray point source}

In gamma-ray astronomy, the ultimate characteristics of a detector is given by the sensitivity to a known point-source standard candle. The capability to detect a photon signal over the background of charged cosmic rays can be expressed through the so-called "signal to noise ratio" $S$ 


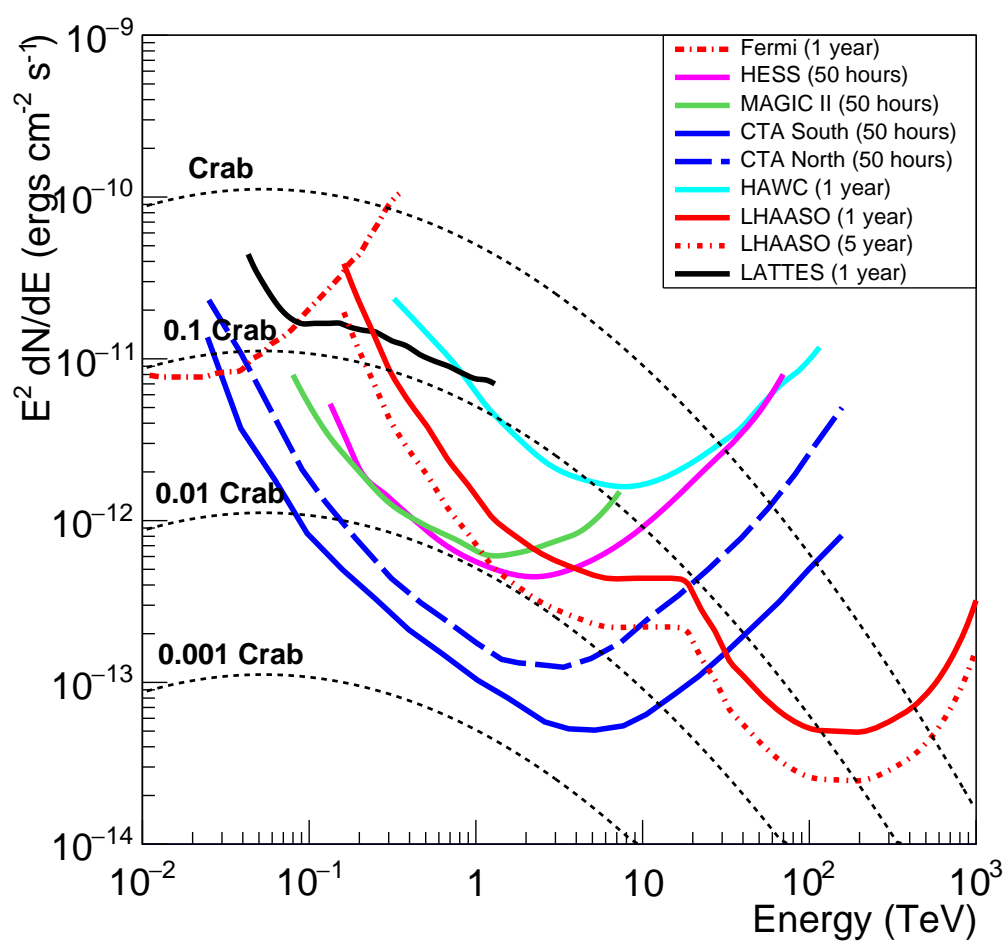

Figure 1: Differential sensitivities to a Crab-like point gamma-ray source of different experiments/projects (multiplied by $\mathrm{E}^{2}$ ). The Crab Nebula spectrum, extrapolated to $1 \mathrm{PeV}$, is reported as a reference together with the spectra corresponding to $10 \%, 1 \%$ and $0.1 \%$ of the Crab flux

$$
S=\frac{\int J_{\gamma}(E) \cdot A_{e f f}^{\gamma}(E) \cdot \varepsilon_{\gamma}(E) \cdot f_{\gamma}(\Delta \Omega) \cdot T d E}{\int J_{b k g}(E) \cdot A_{e f f}^{b k g}(E) \cdot\left(1-\varepsilon_{b k g}(E)\right) \cdot \Delta \Omega \cdot T d E}
$$

where $J_{\gamma}$ and $J_{b k g}$ are the differential fluxes of photon and background, $A_{e f f}^{\gamma}$ and $A_{e f f}^{b k g}$ the effective areas, that determines the number of showers detected in a given observation time $T$, $\Delta \Omega=2 \pi(1-\cos \theta)$ the solid angle around the source and $f_{\gamma}(\Delta \Omega)$ the fraction of $\gamma$-induced showers fitted in the solid angle. The parameters $\varepsilon_{\gamma}$ and $\varepsilon_{b k g}$ are the efficiencies in identifying $\gamma$-induced and background-induced showers, respectively. As most of the parameters are function of the energy, the sensitivity depends on the energy spectra of the cosmic ray background and of the source.

The sensitivity $\mathrm{S}$, formula (2.1), in 1 year can be expressed by

$$
S \propto \frac{\Phi_{\gamma}}{\sqrt{\Phi_{b k g}}} \cdot R \cdot \sqrt{A_{e f f}^{\gamma}} \cdot \frac{1}{\sigma_{\theta}} \cdot Q
$$

where $\Phi_{\gamma}$ and $\Phi_{b k g}$ are the integral fluxes of photon and background, $\sigma_{\theta}$ is the angular resolution, $R=\sqrt{A_{e f f}^{\gamma} / A_{e f f}^{b k g}}$ the $\gamma /$ hadron relative trigger efficiency and $Q=\frac{\varepsilon_{\gamma}}{\sqrt{1-\varepsilon_{b k g}}}$ represents the gain in sensitivity due to the hadron discrimination procedure. Because for the integral fluxes we can 

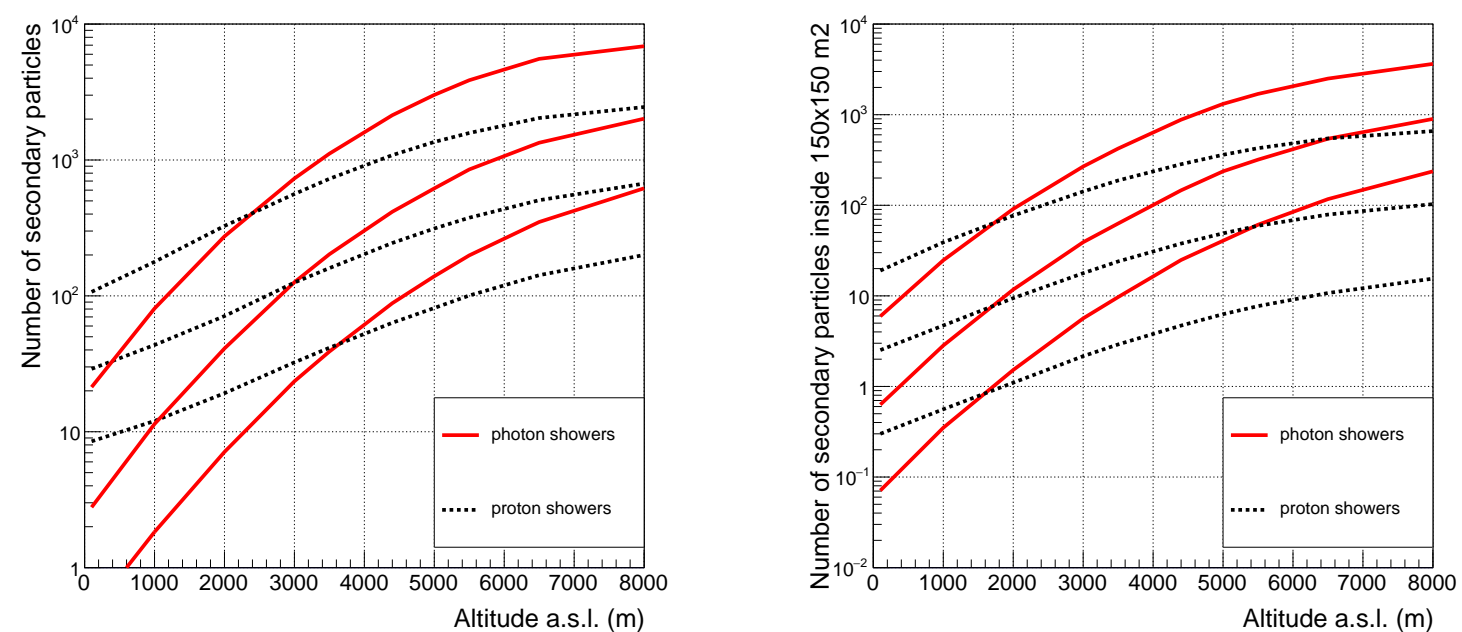

Figure 2: Average number of particles (charged + photons) produced by showers induced by primary photons and protons of different energies at different observation levels. The left plot shows the total size, the right one refers to particles contained inside an area $150 \times 150 \mathrm{~m}^{2}$ centered on the shower core. The plotted energies are 100, 300, $1000 \mathrm{GeV}$ starting from the bottom.

write $\Phi_{\gamma} \sim E_{t h r}^{-\gamma}$ and $\Phi_{b k g} \sim E_{t h r}^{-\gamma_{b k g}}$ we obtain

$$
\frac{\sqrt{\Phi_{b k g}}}{\Phi_{\gamma}} \sim E_{t h r}^{\left(\gamma-\gamma_{b k g} / 2\right)} \sim E_{t h r}^{2 / 3}
$$

being $\gamma \sim 1.5$ and $\gamma_{b k g} \sim 1.7$.

Angular resolution, relative trigger probability, energy threshold and Q-factor are the main parameters, the drives, which determine the sensitivity of a ground-based wide FoV $\gamma$-ray telescope.

\subsection{The energy threshold}

The energy threshold of EAS-arrays is not well defined. In fact, the trigger probability for a shower of a fixed energy increases slowly with energy mainly due to fluctuations in the first interaction height and is not a step function at the threshold energy $E_{t h r}$.

The key to lower the energy threshold is to locate a detector at very high altitude. In the Fig. 2 the average sizes produced by showers induced by primary photons and protons of different energies at different observation levels are plotted. The left plot shows the total number of secondary particles (charged plus photons), the right one shows the number of particles contained inside an area $150 \times 150 \mathrm{~m}^{2}$ centered on the shower core. As can be seen, the number of particles in protoninduced events exceeds the number of particles in $\gamma$-induced ones at low altitudes. This implies that, in gamma-ray astronomy, the trigger probability is higher for the background than for the signal.

The small number of charged particles in sub-TeV showers within $150 \mathrm{~m}$ from the core imposes to locate experiments at extreme altitudes ( $>4500 \mathrm{~m}$ asl). At $5500 \mathrm{~m}$ asl $100 \mathrm{GeV} \gamma$-induced showers contains about 8 times more particles than proton showers within $150 \mathrm{~m}$ from the core. This fact can be appreciated in the Fig. 3 where the ratio of particle numbers (charged + photons) 

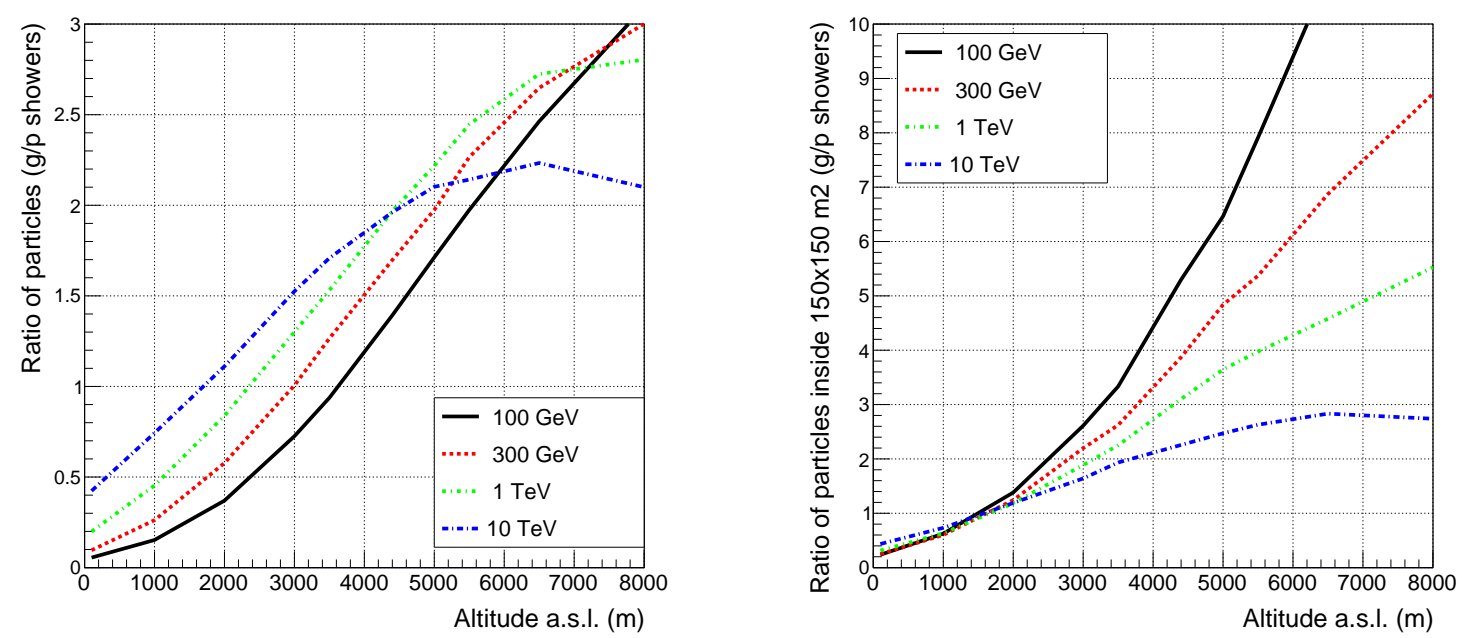

Figure 3: Ratio of particle numbers (charged + photons) in photon- and proton-induced showers of different energies as a function of the observation level. The left plot shows the total size, the right one refers to particles contained inside an area $150 \times 150 \mathrm{~m}^{2}$ centered on the shower core.

in photon- and proton-induced showers of different energies as a function of the observation level are shown.

\subsection{Relative Trigger Efficiency $R$}

The effective area $A_{e f f}$ is mainly a function of the number of charged particles at the observation level, the dimension and coverage of the detector and the trigger logic. Moving a given detector at different altitudes $A_{\text {eff }}$ is proportional to number of charged particle.

The Fig. 3 shows the ratio $\mathrm{R}=\mathrm{N}^{\gamma} / \mathrm{N}^{p}$ of all secondary particles (charged plus photons) in photon- and proton-induced showers as a function of the altitude. The left plot refers to the total size, the right one to particles contained inside an area $150 \times 150 \mathrm{~m}^{2}$ centered on the shower core.

When $\mathrm{R}<1$ the trigger probability is higher for protons than for photons. On the contrary, when $\mathrm{R}>1$ the background of cosmic rays is reduced by the different trigger efficiency and an intrinsic $\gamma /$ hadron discrimination enriches the selected sample by photons.

Being $\mathrm{R}$ proportional to the ratio of effective areas an altitude $>4500 \mathrm{~m}$ asl is required to increase the sensitivity of a gamma-ray telescope in the hundreds $\mathrm{GeV}$ energy range.

Comparing the two plots of Fig. 3 we can see that, as expected, the $\gamma$-showers show a more compact particle distribution at the observation level. Therefore, at extreme altitudes the trigger efficiency of photon event at hundreds $\mathrm{GeV}$ is highly favoured if we consider only secondary particles within $150 \mathrm{~m}$ from the core.

Showers of all energies have the same slope well after the shower maximum: $\approx 1.65 \mathrm{x}$ decrease per radiation length (r.l.) [?]. This implies that if a given detector is located one radiation length higher in atmosphere, the result will be $\mathrm{a} \approx 1.65 \mathrm{x}$ decrease of the energy threshold.

But the energy threshold is also a function of the detection medium and of the coverage, the ratio between the detector and instrumented areas. Classical EAS arrays are constituted by a large number of detectors (typically plastic scintillators) spread over an area of order of $10^{4}-10^{5} \mathrm{~m}^{2}$ 


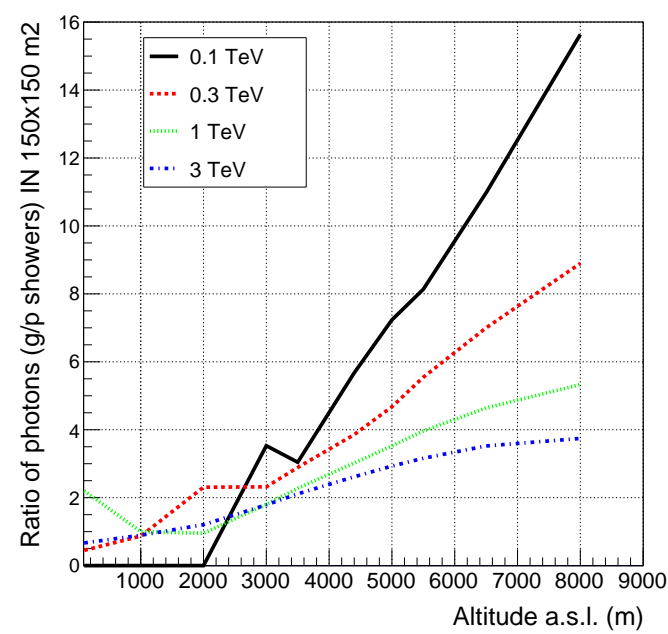

Figure 4: Ratio of secondary photons in gammaand proton-induced showers of different energies as a function of the observation level. The particles have been selected inside an area $150 \times 150 \mathrm{~m}^{2}$ centered on the shower core.

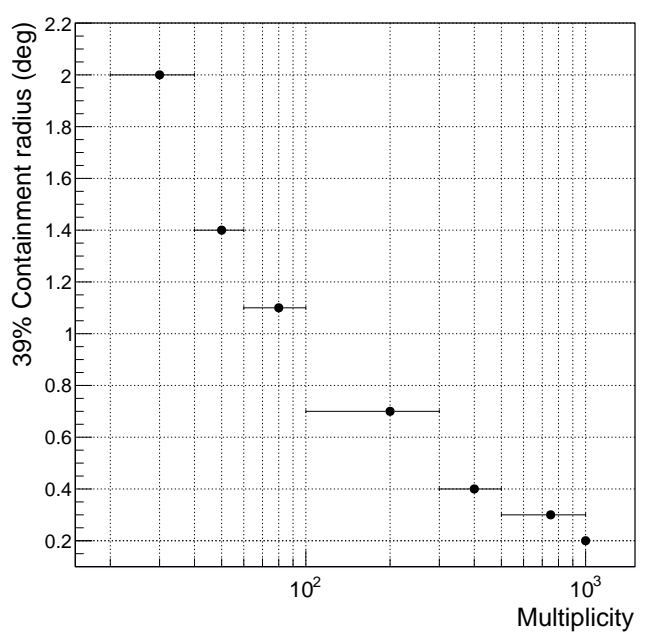

Figure 5: Angular resolution (defined as the 39\% containment radius) of a $100 \times 100 \mathrm{~m}^{2}$ ARGO-like RPC carpet operated at $5500 \mathrm{~m}$ asl as a function of the number of fired pads.

with a coverage factor of about $10^{-3}$. This poor coverage limits the energy threshold because small low-energy showers cannot be efficiently triggered by a sparse array.

To exploit the potential of the coverage, a high granularity of the read-out must be coupled to image the shower front with high resolution. As will be discussed in the following, the combination of high coverage and high granularity of the read-out improves also the angular resolution providing more detailed informations about the shower temporal profile.

Another important factor to lower the energy threshold of a detector is the secondary photon component detection capability. Gamma rays dominate the shower particles on ground: at $4300 \mathrm{~m}$ asl a $100 \mathrm{GeV}$ photon-induced shower contains on average 7 times more secondary photons than electrons [3]. In $\gamma$-showers the ratio $\mathrm{N}_{\gamma} / \mathrm{N}_{c h}$ decreases if the comparison is restricted to a small area around the shower core. For instance, we get $\mathrm{N}_{\gamma} / \mathrm{N}_{c h} \sim 3.5$ at a distance $\mathrm{r}<50 \mathrm{~m}$ from the core for $100 \mathrm{GeV}$ showers [3].

In Fig. 4 the ratio of secondary photons within $150 \mathrm{~m}$ from the shower core for gamma- and proton-induced showers of different energies is plotted as a function of the altitude. The number of secondary photons in low energy $\gamma$-showers exceeds by large factors the number of gammas in p-showers with increasing altitude. Detecting photons at extreme altitudes provides an intrinsic $\gamma /$ hadron separation tool.

\section{The angular resolution}

In a search for cosmic point $\gamma$-ray sources with ground-based arrays the main problem is the rejection of the background due to charged cosmic rays, therefore a good angular resolution (i.e., the accuracy in estimating the arrival direction) is fundamental. 
The usual method for reconstructing the shower direction is performing a $\chi^{2}$ fit to the recorded arrival times $t_{i}$ by minimization of

$$
\chi^{2}=\sum_{i} w\left(f-t_{i}\right)^{2}
$$

where the sum includes all detectors with a time signal. Usually the function $f$ describes a plane, a cone with a fixed cone slope or a plane with curvature corrections as a function of core distance $r$ and multiplicity $m$. Fitted are a time offset and the two direction cosines. The weights $w$ are generally chosen to be an empirical function of the number $m$ of particles registered in a counter, a function of $r$ or a function of $r$ and $m$. This represents in general terms the usual fitting procedure of the "time of flight" technique. Improvement to this scheme can be achieved by excluding from the analysis the time values belonging to the non-gaussian tails of the arrival time distributions by performing some successive $\chi^{2}$ minimizations for each shower $[4,5]$. In fact, the distribution of the arrival times shows non-Gaussian tails at later times, mainly due to multiple scattering of low energy electrons but also to incorrect counters calibrations and to random coincidences. These non-Gaussian tails are expected typically to be $20 \%$ of all measured time values.

Placing a thin sheet of converter above the detector can improve the angular resolution due to, qualitatively: (1) absorption of low energy electrons (and photons) which no longer contribute to the time signal; (2) multiplication process of high-energy electrons (and photons) which produce an enhancement of the signal. The enhanced signal reduces the timing fluctuations: the contributions gained are concentrated near the ideal time profile because the high energy particles travel near the front of the shower while those lost tend to lag far behind.

The angular resolution of a $100 \times 100 \mathrm{~m}^{2}$ ARGO-like RPC carpet [2] operated at $5500 \mathrm{~m}$ asl is shown in the Fig. 5 as a function of the number of fired pads. In this calculation we used the reconstruction procedure used in ARGO-YBJ and described in [5], not optimized yet for a larger carpet operated at higher altitude.

To describe the PSFs analytically, for small values of fired pads that cannot be simply fitted by a two-dimensional Gaussian function, the simulated distributions have been fitted with a linear combination of two Gaussians. In general, when the PSF is described by a single Gaussian $(F(r)=$ $1 /\left(2 \pi \sigma^{2}\right) \exp \left(-r^{2} / \sigma^{2}\right)$, where $r$ is the angular distance from the source position), the value of the root mean square $\sigma$ is commonly defined as the "angular resolution". In this case, the fraction of events within $1 \sigma$ is $39 \%$. For our PSFs, the value of the $39 \%$ containment radius $R_{39}$ ranges from $0.2^{\circ}$ for $\mathrm{N}_{\text {pad }} \geq 1000$ to about $2^{\circ}$ for $\mathrm{N}_{\text {pad }}=20-39$.

\section{Conclusions}

In the next decade CTA-North and LHAASO are expected to be the most sensitive instruments to study Gamma-Ray Astronomy in the Northern hemisphere from about $20 \mathrm{GeV}$ up to PeV. A new wide FoV telescope to study the $\gamma$-sky of the Southern hemisphere above $100 \mathrm{GeV}$ with high sensitivity and to continuously monitor the Galactic Center and the Inner Galaxy is a high priority. To be useful to CTA-South such a detector should have a sensitivity of a few percent of the Crab Nebula flux in the $100 \mathrm{GeV}$ energy range.

Extreme altitude (above $5000 \mathrm{~m}$ asl), high coverage and high efficiency in the conversion of secondary photons are the key to improve the angular resolution and the sensitivity to gamma-ray 
sources. The discrimination of the background of charged cosmic rays in the sub-TeV/TeV energy region is very challenging due to the very small event multiplicity and to the absence of secondary muons. It is necessary to perform calculations to evaluate the background rejection capability.

\section{References}

[1] P. Assis et al., arXiv:1607.03051.

[2] G. Di Sciascio, Int. J. of Mod. Phys. D23 (2014) 1430019.

[3] G. Di Sciascio et al., Astrop. Phys. 6 (1997) 313.

[4] G. Di Sciascio et al., Proc. of the 29th International Cosmic Ray Conference (ICRC 05), Pune, India (Tata Institute of Fundamental Research, Mumbai, India, 2005), Vol. 6, p. 33.

[5] B. Bartoli et al., PRD 84 (2011) 022003. 